\title{
Sulla stima della vita a fatica di giunti saldati soggetti a carichi multiassiali ad ampiezza variabile
}

\author{
L. Susmel \\ Dipartimento di Ingegneria, Università di Ferrara, Ferrara (Italia); ssl@unife.it \\ Department of Mechanical Engineering, Trinity College, Dublin (Ireland) \\ R. Tovo \\ Dipartimento di Ingegneria, Università di Ferrara, Ferrara (Italia) \\ D. Benasciutti \\ Dipartimento di Ingegneria Elettrica Gestionale Meccanica, Università di Udine, Udine, (Italy)
}

RIASSUNTO. Nel presente articolo viene proposta una nuova metodologia di progettazione a fatica, basata sull'utilizzo del metodo delle Curve di Wöhler Modificate, per la previsione della vita a fatica di giunzioni saldate, sia in acciaio che in alluminio, soggette a carichi multiassiali ad ampiezza variabile. In particolare, il criterio delle Curve di Wöhler Modificate è stato applicato determinando l'orientazione del piano critico mediante il Metodo della Massima Varianza, ovvero definendo il piano critico come quello contenente la direzione che sperimenta la massima varianza della tensione tangenziale risolta. L'accuratezza della metodologia di progettazione a fatica proposta nella presente memoria è stata valutata mediante due serie di dati sperimentali di letteratura ottenute sollecitando, sia ad ampiezza costante che variabile, giunti saldati tubo-piastra in acciaio e lega di alluminio con carichi di flesso/torsione in fase e sfasati di $90^{\circ}$. Il criterio delle Curve di Wöhler Modificate, applicato in concomitanza con il Metodo della Massima Varianza, si è dimostrato capace di fornisce stime accurate della durata a fatica anche in presenza di sollecitazioni multiassiali ad ampiezza variabile, e questo sia quando applicato in termini di tensioni nominali che in termini di tensioni di "hot-spot".

ABSTRACT. This paper summarises an attempt of devising a new engineering approach, based on the so-called Modified Wöhler Curve Method, suitable for estimating fatigue lifetime of both steel and aluminium welded connections subjected to variable amplitude multiaxial fatigue loading. The Maximum Variance Method is used to determine the orientation of the critical plane and the cycle counting is directly performed in terms of shear stress resolved along the maximum variance direction. The accuracy of the proposed methodology was checked by using two different datasets taken from the literature and generated by testing both steel and aluminium tube-to-plate welded connections under in-phase and $90^{\circ}$ out-of-phase variable amplitude bending and torsion. The proposed fatigue life assessment technique was seen to be highly accurate, resulting in estimates falling within the scatter bands of the curves used to calibrate the Modified Wöhler Curve Method itself. This seems to strongly support the idea that the proposed approach can be considered as an effective engineering tool, capable of performing multiaxial fatigue assessment under variable amplitude loading by fully complying with recommendations of the available standard codes.

KEYwORDS. Multiaxial Fatigue; Variable amplitude; Weldments. 


\section{INTRODUZIONE}

$\mathrm{L}$

e strutture saldate sono spesso soggette in esercizio a stati di sollecitazione e/o deformazione multiassiali, responsabili di un danneggiamento in prossimità del cordone di saldatura. Fin dalla metà del secolo scorso un'intensa attività di ricerca si è specificatamente rivolta alla formulazione di criteri di calcolo, basati sull'utilizzo di tensioni nominali, strutturali o locali [1-3], applicabili a giunti saldati soggetti a sollecitazioni multiassiali ad ampiezza costante. Tuttavia, esistono solo alcuni approcci specificatamente formalizzati per stimare la resistenza a fatica di giunzioni saldate soggette a sollecitazioni multiassiali ad ampiezza variabile [4-7]. Riguardo a questo aspetto, è interessante notare che, per sollecitazioni ad ampiezza variabile, alcune normative propongono procedure di calcolo essenzialmente basate sulla combinazione di tensioni normali e tangenziali, e sull'applicazione dell'ipotesi di Palmgren-Miner, lasciando tuttavia aperta la necessità di una sistematica indagine e verifica sperimentale [8-10].

Vista l'importanza di sviluppare metodologie di calcolo efficienti ed accurate utilizzabili nella pratica progettuale per valutare la resistenza a fatica di giunzioni saldate, questo lavoro intende proporre un approccio di piano critico basato sull'uso delle Curve di Wöhler Modificate (CWM) [11-14] applicabile a sollecitazioni multiassiali ad ampiezza variabile.

In particolare, l'approccio proposto utilizza congiuntamente il metodo delle CWM ed il Metodo della Massima Varianza (MMV) [15, 16], per stimare la vita a fatica post-processando lo stato di sollecitazione agente sul piano critico, dove la tensione tangenziale viene risolta lungo la sua direzione di massima varianza. L'aspetto più interessante dell'approccio proposto è la possibilità di applicare i metodi di conteggio convenzionali alla tensione tangenziale risolta sulla direzione di massima varianza, estendendo di fatto l'applicabilità dei metodi di calcolo uniassiali a stati multiassiali di tensione.

E' importante, infine, evidenziare come l'accuratezza del metodo discusso nel presente articolo è stata verificata considerando dati sperimentali relativi a giunti saldati tubo-piastra in acciaio e lega di alluminio, soggetti ad uno spettro di carico di flesso-torsione in fase ed in quadratura.

\section{METODO DELLE CURVE DI WÖHLER MODIFICATE}

$\mathrm{N}$ el metodo delle CWM si assume che la probabilità di nucleazione della cricca di fatica sia massima sul piano dove è massima la variazione della tensione tangenziale, $\Delta \tau$. La complessità dello stato di sollecitazione sul piano critico è poi quantificata combinando la variazione della tensione tangenziale, $\Delta \tau$, e normale, $\Delta \sigma_{\mathrm{n}}$, tramite il seguente indice $[11,12]$ :

$$
\rho_{\mathrm{w}}=\frac{\Delta \sigma_{\mathrm{n}}}{\Delta \tau},
$$

che risulta essere unitario per sollecitazioni monoassiali con $\mathrm{R}=-1$, mentre è uguale a zero per sollecitazioni di torsione alterna. Il parametro $\varrho_{w}$, inoltre, è correlato al livello di non-proporzionalità della sollecitazione applicata, mentre il suo valore non dipende dalla presenza di tensioni medie non nulle, e questo in accordo con quanto suggerito dalle procedure proposte nelle normative vigenti per giunti non trattati termicamente [8-10].

La stima della vita a fatica in accordo con il metodo delle CWM è illustrata nel diagramma di Wöhler modificato di Fig. 1a, che riporta le rette che forniscono il numero di cicli a rottura, $\mathrm{N}_{\mathrm{f}}$, in funzione della variazione della tensione tangenziale, $\Delta \tau$, per differenti valori di $\varrho_{\mathrm{w}}$. In particolare, al diminuire di $\varrho_{\mathrm{w}}$, le rette traslano verso l'alto, indicando una diminuzione del danno a fatica. In altri termini, il metodo delle CWM assume che, per un dato materiale, il danneggiamento a fatica dipenda esclusivamente dai valori di $\Delta \tau$ e $\rho_{\mathrm{w}}$, indipendentemente dalla complessità del percorso di carico individuato sul piano critico. Ogni curva di Wöhler è poi caratterizzata dalla sua pendenza inversa, $\mathrm{k}_{\tau}$, e dall'ampiezza di riferimento, $\Delta \tau_{A, \text { Ref, }}$ estrapolata a $\mathrm{N}_{\mathrm{A}}$ cicli a rottura (in accordo con le vigenti normative [8-10], si assume $\mathrm{N}_{\mathrm{A}}=2 \cdot 10^{6}$ cicli a rottura).

Il diagramma di Wöhler modificato di Fig. 1 permette il calcolo della vita a fatica una volta stimate su base sperimentale le correlazioni fra $\Delta \tau_{\mathrm{A}, \mathrm{Ref}}$ e $\varrho_{\mathrm{w}}$ e quella fra $\mathrm{k}_{\tau}$ e $\varrho_{\mathrm{w}}$. In particolare, adottando una semplice dipendenza lineare è possibile calcolare le costanti sulla base delle curve a fatica a trazione $\left(\varrho_{\mathrm{w}}=1\right)$ e a torsione $\left(\varrho_{\mathrm{w}}=0\right)$ alterne $[11,12]$ :

$$
\mathrm{k}_{\tau}\left(\rho_{\mathrm{w}}\right)=\left(\mathrm{k}-\mathrm{k}_{0}\right) \rho_{\mathrm{w}}+\mathrm{k}_{0} \quad \text { per } \rho_{\mathrm{w}} \leq \rho_{\mathrm{w}, \lim }
$$




$$
\begin{array}{ll}
\mathrm{k}_{\tau}\left(\rho_{\mathrm{w}}\right)=\mathrm{k}_{\tau}\left(\rho_{\mathrm{w}, \lim }\right)=\mathrm{const} & \text { per } \rho_{\mathrm{w}}>\rho_{\mathrm{w}, \lim } \\
\Delta \tau_{\mathrm{A}, \operatorname{Ref}}\left(\rho_{\mathrm{w}}\right)=\left(\frac{\Delta \sigma_{\mathrm{A}}}{2}-\Delta \tau_{\mathrm{A}}\right) \rho_{\mathrm{w}}+\Delta \tau_{\mathrm{A}} & \text { per } \rho_{\mathrm{w}} \leq \rho_{\mathrm{w}, \lim } \\
\Delta \tau_{\mathrm{A}, \operatorname{Ref}}\left(\rho_{\mathrm{w}}\right)=\Delta \tau_{\mathrm{A}, \operatorname{Ref}}\left(\rho_{\mathrm{w}, \lim }\right)=\mathrm{const} & \text { per } \rho_{\mathrm{w}}>\rho_{\mathrm{w}, \lim }
\end{array}
$$

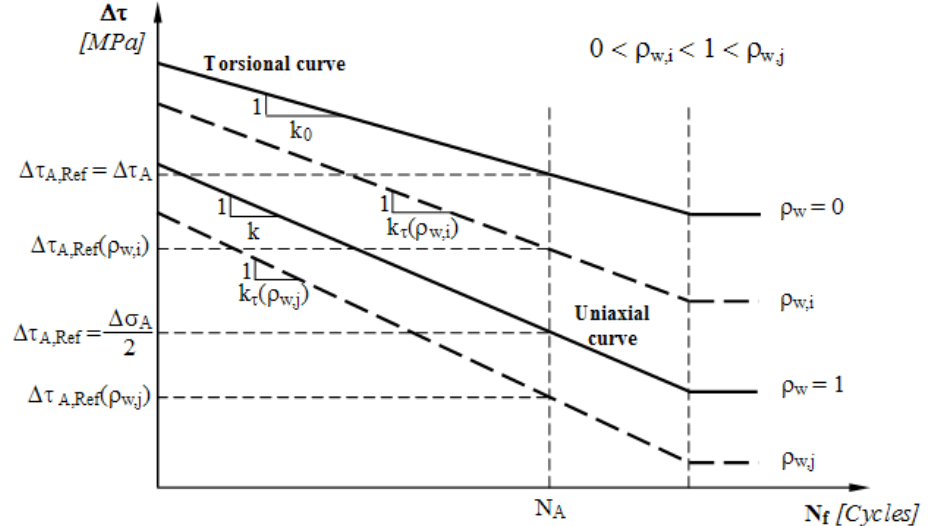

(a)

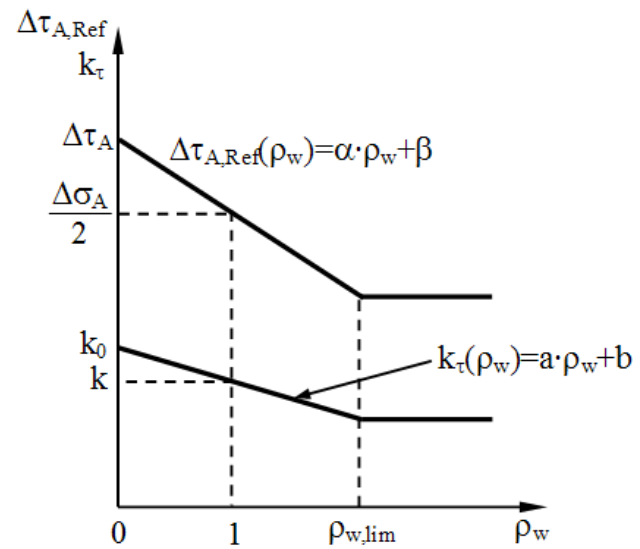

(b)

Figura 1: (a) Diagramma di Wöhler modificato e (b) andamento di $\Delta \tau_{\mathrm{A}, \mathrm{Ref}}$ e $\mathrm{k}_{\tau}$ con $\varrho_{\mathrm{w}}$.

Il significato dei simboli e l'andamento qualitativo delle funzioni è mostrato in Fig. 1b. Il precedente diagramma schematico mostra anche l'esistenza di un valore soglia, $\varrho_{\mathrm{w}, \mathrm{lim}}$, oltre il quale si assume che $\Delta \tau_{\mathrm{A}, \mathrm{Ref}}$ e $\mathrm{k}_{\tau}$ restino entrambi costanti. In particolare, numerosi risultati sperimentali confermano che per elevati valori di $\varrho_{w}$ gli approcci di piano critico tendono a fornire stime troppo conservative, e questo sembra essere dovuto al fatto che, in tali circostanze, il danneggiamento a fatica non è solo correlato alle tensioni tangenziali, ma anche, e in modo molto forte, a quelle normali al piano critico. Questo implica che, per valori di $\varrho_{w}$ elevati, stime accurate possano essere ottenute solo valutando l'influenza delle tensioni normali al piano critico mediante differenti assunzioni. In particolare, si è osservato che in componenti non intagliati $\varrho_{w, l i m}$ è correlato alle proprietà a fatica del materiale ad alto numero di cicli [14, 17], mentre in componenti intagliati (analizzati in termini di tensioni nominali) $\varrho_{w}$ lim è più difficilmente quantificabile, dato che le tensioni sopraccitate hanno scarsa correlazione con i fenomeni fisici responsabili della fase di nucleazione della cricca di fatica. Quando il metodo delle CWM viene utilizzato per stimare la vita a fatica in componenti saldati, si suggerisce sempre l'utilizzo di valori di $\varrho_{\mathrm{w}, \text { lim }}$ compresi nell'intervallo $1.4 \div 1.5$, e questo valutando lo stato tensionale sia in termini di quantità nominali, che in termini di grandezze strutturali.

\section{Metodo Della Massima VARIANZa}

I

n sollecitazioni random uniassiali, sia gaussiane che non-gaussiane, il danno a fatica è proporzionale alla varianza [1821]. Il Metodo della Massima Varianza (MMV) assume che il piano di massimo danneggiamento a fatica coincida con il piano contenente la direzione dove la tensione tangenziale risolta ha varianza massima [14-16].

Con riferimento a Fig. 2, è assegnato il piano $\Delta$ di normale $\overline{\mathrm{n}}$, su cui sono individuati i versori $\overline{\mathrm{a}}$ e $\overline{\mathrm{b}}$, caratterizzati, nel sistema di riferimento Oxyz, dai seguenti coseni direttori:

$$
\overline{\mathrm{n}}=\left[\begin{array}{c}
\mathrm{n}_{\mathrm{x}} \\
\mathrm{n}_{\mathrm{y}} \\
\mathrm{n}_{\mathrm{z}}
\end{array}\right]=\left[\begin{array}{c}
\sin (\theta) \cos (\phi) \\
\sin (\theta) \sin (\phi) \\
\cos (\theta)
\end{array}\right], \overline{\mathrm{a}}=\left[\begin{array}{c}
\mathrm{a}_{\mathrm{x}} \\
\mathrm{a}_{\mathrm{y}} \\
\mathrm{a}_{\mathrm{z}}
\end{array}\right]=\left[\begin{array}{c}
\sin (\phi) \\
-\cos (\phi) \\
0
\end{array}\right], \overline{\mathrm{b}}=\left[\begin{array}{c}
\mathrm{b}_{\mathrm{x}} \\
\mathrm{b}_{\mathrm{y}} \\
\mathrm{b}_{\mathrm{z}}
\end{array}\right]=\left[\begin{array}{c}
\cos (\theta) \cos (\phi) \\
\cos (\theta) \sin (\phi) \\
-\sin (\theta)
\end{array}\right]
$$


l'orientazione di una generica retta $m$ appartenente al piano $\Delta$ è individuata dall'angolo $\xi$ :

$$
\overline{\mathrm{m}}=\left[\begin{array}{c}
\mathrm{m}_{\mathrm{x}} \\
\mathrm{m}_{\mathrm{y}} \\
\mathrm{m}_{\mathrm{z}}
\end{array}\right]=\left[\begin{array}{c}
\cos (\xi) \sin (\phi)+\sin (\xi) \cos (\theta) \cos (\phi) \\
-\cos (\xi) \cos (\phi)+\sin (\xi) \cos (\theta) \sin (\phi) \\
-\sin (\xi) \sin (\theta)
\end{array}\right]
$$

La tensione tangenziale $\tau_{\mathrm{m}}(\mathrm{t})$ risolta lungo $\overline{\mathrm{m}}$ può pertanto essere calcolata come:

$$
\overline{\mathrm{m}}=\left[\begin{array}{c}
\mathrm{m}_{\mathrm{x}} \\
\mathrm{m}_{\mathrm{y}} \\
\mathrm{m}_{\mathrm{z}}
\end{array}\right]=\left[\begin{array}{c}
\cos (\xi) \sin (\phi)+\sin (\xi) \cos (\theta) \cos (\phi) \\
-\cos (\xi) \cos (\phi)+\sin (\xi) \cos (\theta) \sin (\phi) \\
-\sin (\xi) \sin (\theta)
\end{array}\right]
$$

Definendo ora il vettore delle tensioni come:

$$
\bar{s}(t)=\left[\begin{array}{llllll}
\sigma_{x}(t) & \sigma_{y}(t) & \sigma_{z}(t) & \tau_{x y}(t) & \tau_{x z}(t) & \tau_{y z}(t)
\end{array}\right]
$$

la tensione tangenziale risolta lungo $\overline{\mathrm{m}}$ può essere direttamente calcolata a mezzo del seguente prodotto scalare:

$$
\tau_{\mathrm{m}}(\mathrm{t})=\overline{\mathrm{d}} \bullet \overline{\mathrm{s}}(\mathrm{t}),
$$

dove $\overline{\mathrm{d}}$ è un vettore dei coseni direttori di facile determinazione (si omette l'espressione).

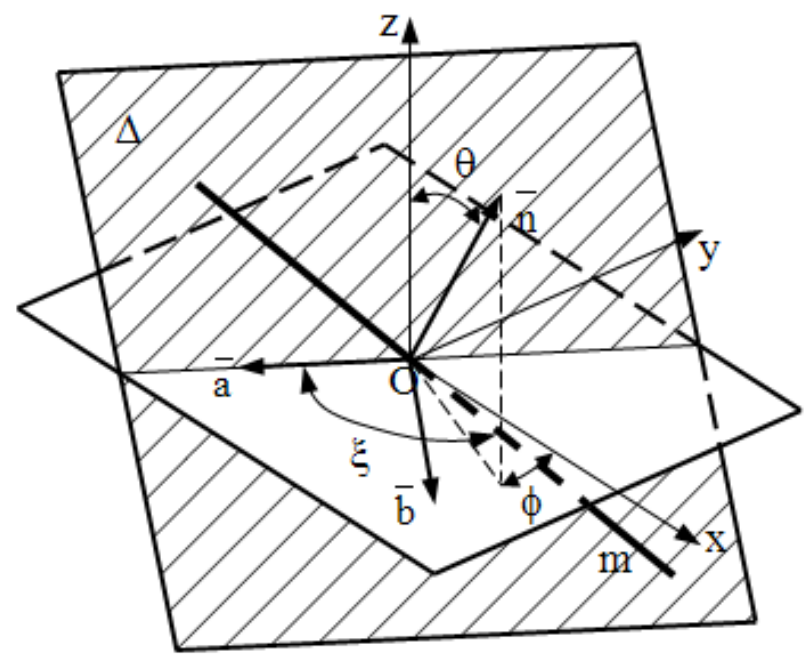

Figura 2: Definizione delle grandezze utilizzate dal MMV.

In termini generali, la varianza della tensione tangenziale risolta $\tau_{\mathrm{m}}(\mathrm{t})$ può scriversi come:

$$
\operatorname{Var}\left[\tau_{m}(t)\right]=\operatorname{Var}\left[\sum_{k} d_{k} s_{k}(t)\right]=\sum_{i} \sum_{j} d_{i} d_{j} \operatorname{Cov}\left[s_{i}(t), s_{j}(t)\right]
$$

dove per $i=j$ si ottengono termini di varianza, $\operatorname{Cov}\left[\mathrm{s}_{\mathrm{i}}(\mathrm{t}), \mathrm{s}_{\mathrm{j}}(\mathrm{t})\right]=\operatorname{Var}\left[\mathrm{s}_{\mathrm{i}}(\mathrm{t})\right]$, mentre per $i \neq j$ si hanno termini di covarianza. Definendo, infine, la matrice di covarianza come:

$$
\mathrm{C}_{\mathrm{ij}}=\operatorname{Cov}\left[\mathrm{s}_{\mathrm{i}}(\mathrm{t}), \mathrm{s}_{\mathrm{j}}(\mathrm{t})\right]
$$

è possibile riscrivere formalmente l'Eq. (8) in modo compatto, ovvero: 


$$
\operatorname{Var}\left[\tau_{\mathrm{m}}(\mathrm{t})\right]=\overline{\mathrm{d}}^{\mathrm{T}}[\mathrm{C}] \overline{\mathrm{d}}
$$

Nell'ipotesi che il piano critico contenga la direzione dove $\tau_{\mathrm{m}}(\mathrm{t})$ ha varianza massima, è immediato utilizzare la Eq. (10) per individuare tale piano mediante i coseni direttori rappresentati dal vettore $\overline{\mathrm{d}}$, e calcolare così in modo diretto anche le grandezze ad esso correlate.

\section{ANALISI DI SOLLECITAZIONI MULTIASSIALI AD AMPIEZZA VARIABILE}

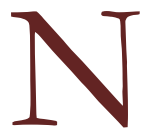

oti i vettori $\overline{\mathrm{n}}, \overline{\mathrm{m}}$ che definiscono il piano critico e la direzione di massima varianza, è possibile ricondurre una storia temporale del tensore delle tensioni alle storie di carico della tensione tangenziale risolta, $\tau(\mathrm{t})$, e della tensione normale al piano critico, $\sigma_{\mathrm{n}}(\mathrm{t})$. Questa semplificazione permette, pertanto, di estendere i metodi di conteggio monoassiali (es. rainflow) al caso multiassiale.

In particolare, per la semplice storia temporale in Fig. 3a è immediato individuare per ogni ciclo i range della tensione tangenziale, $\Delta \tau(\mathrm{t})$, e normale, $\Delta \sigma_{\mathrm{n}}(\mathrm{t})$, e quindi calcolare l'indice $\rho_{\mathrm{w}, \mathrm{i}}, \rho_{\mathrm{w}, j}, \ldots$, con cui determinare, attraverso le relazioni (2), le corrispondenti curve di resistenza. Per ogni valore di $\rho_{\mathrm{w}}$, ed individuato lo spettro di carico, si può quindi calcolare prima il danno di ogni singolo ciclo contato, poi il danno totale nell'ipotesi di Palmgren-Miner, ovvero:

$$
\mathrm{D}_{\mathrm{i}}=\sum \frac{\mathrm{n}}{\mathrm{N}_{\mathrm{f}}\left(\rho_{\mathrm{w}, \mathrm{i}}\right)} \quad, \quad \mathrm{D}_{\mathrm{j}}=\sum \frac{\mathrm{n}}{\mathrm{N}_{\mathrm{f}}\left(\rho_{\mathrm{w}, \mathrm{j}}\right)} \quad, \quad \ldots \quad \mathrm{D}_{\mathrm{tot}}=\ldots+\mathrm{D}_{\mathrm{i}}+\mathrm{D}_{\mathrm{j}}+\ldots
$$

dove i cicli a rottura $\mathrm{N}_{\mathrm{f}}\left(\rho_{\mathrm{w}, \mathrm{i}}\right), \mathrm{N}=\left(\rho_{\mathrm{w}, \mathrm{j}}\right)$ si valutano sull'opportuna curva di Wöhler modificata, individuata dal particolare valore di $\rho_{\mathrm{w}}$ considerato (vedere Fig. 1).

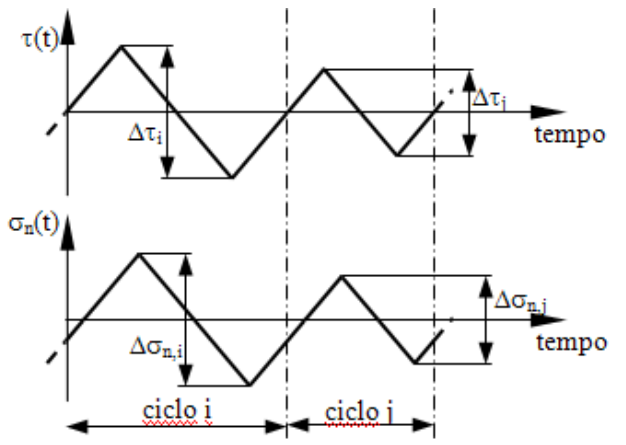

(a)

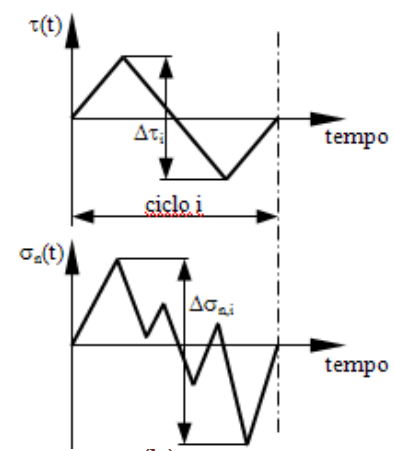

(b)

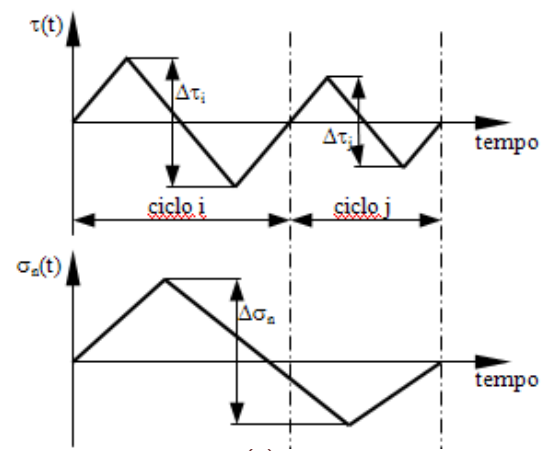

(c)

Figura 3: (a) Esempio di conteggio per una storia di carico semplice;

(b), (c) esempi di conteggio per storie di carico più complesse.

L'esempio di Fig. 3a, in quanto molto semplice, permette l'individuazione univoca dei cicli. Per sollecitazioni più complesse, invece, il conteggio dei cicli può risultare non solo poco agevole ma anche ambiguo. In Fig. 3b, per esempio, ad un singolo ciclo della tensione $\tau(\mathrm{t})$ sono associati più cicli di $\sigma_{\mathrm{n}}(\mathrm{t})$, così che la definizione di $\Delta \sigma_{\mathrm{n}}(\mathrm{t})$ non è evidentemente univoca. In modo simile, in Fig. $3 \mathrm{c}$ un singolo ciclo di ampiezza $\Delta \sigma_{\mathrm{n}}(\mathrm{t})$ caratterizza più cicli della tensione $\tau(\mathrm{t})$, rendendo difficile il calcolo del corrispondente $\rho_{\mathrm{w}}$ per ogni ciclo in $\tau(\mathrm{t})$ contato. Questo suggerisce che ulteriori studi di tipo teorico/sperimentale debbano essere condotti onde formalizzare delle procedure che consentano di eseguire il conteggio dei cicli anche in presenza di storie di carico multiassiali estremamente complesse.

\section{CONFRONTO CON I DATI SPERIMENTALI}

prescindere dai problemi ancora aperti menzionati alla fine del precedente paragrafo, la precisione del metodo proposto è stata verificata utilizzando i dati sperimentali riportati in $[5,6]$ e ottenuti sollecitando, sia ad ampiezza costante che variabile, giunti saldati tubo-piastra in acciaio e lega di alluminio, con carichi di flesso-torsione in 
fase e sfasati di $90^{\circ}$ (dove il rapporto fra l'ampiezza della tensione tangenziale nominale rispetto alla tensione nominale flessionale era costante ed uguale a 0.58). In altre parole, da un punto di vista del conteggio dei cicli, il metodo proposto è stato valutato con storie di carico del tipo riportato in Fig. 3a, ovvero in condizioni in cui il conteggio dei cicli in $\tau(t)$ e la determinazione del corrispondente valore di $\rho_{\mathrm{w}}$ è univoca.

Per sollecitazioni di sola flessione o torsione ad ampiezza costante, la Fig. 4 mostra come le curve fornite dall'IIW [10], riferite alle tensioni nominali, forniscano, in genere, stime conservative, aspetto peraltro confermato dai valori riportati in Tab. 1, dove le curve sperimentali (per un livello di confidenza del 95\%), espresse come resistenza a $\mathrm{N}_{\mathrm{A}}=2 \cdot 10^{6}$ cicli a rottura e pendenza inversa $\mathrm{k}$, sono confrontate con i corrispondenti valori delle curve di normativa. I dati sperimentali ad ampiezza variabile qui utilizzati per validare il metodo proposto considerano uno spettro di carico gaussiano di $5 \cdot 10^{4}$ cicli complessivi (si veda $[5,6])$.

\begin{tabular}{|c|c|c|c|c|c|c|c|c|c|c|c|c|c|c|c|c|}
\hline \multirow[t]{2}{*}{ Materiale } & \multicolumn{4}{|c|}{ Risultati sperimentali } & \multicolumn{6}{|c|}{ Tensioni nominali } & \multicolumn{6}{|c|}{ Tensioni strutturali ('hot-spot') } \\
\hline & $\begin{array}{c}\Delta \sigma_{\mathrm{A}, 50 \%} \% \\
{[\mathrm{MPa}]}\end{array}$ & $\mathrm{k}$ & $\begin{array}{c}\Delta \tau_{\mathrm{A}, 50 \%} \\
{[\mathrm{MPa}]}\end{array}$ & $\mathrm{k}_{0}$ & $\begin{array}{c}\Delta \sigma_{\mathrm{A}} \\
{[\mathrm{MPa}]}\end{array}$ & $\begin{array}{c}\Delta \sigma_{\mathrm{A}, 50 \%} \\
{[\mathrm{MPa}]}\end{array}$ & $\mathrm{k}$ & $\begin{array}{c}\Delta \tau_{\mathrm{A}} \\
{[\mathrm{MPa}]}\end{array}$ & $\begin{array}{c}\Delta \tau_{\mathrm{A}, 50 \%} \\
{[\mathrm{MPa}]}\end{array}$ & $\mathrm{k}_{0}$ & $\begin{array}{c}\Delta \sigma_{\mathrm{A}} \\
{[\mathrm{MPa}]}\end{array}$ & $\begin{array}{c}\Delta \sigma_{\mathrm{A}, 50 \%} \\
{[\mathrm{MPa}]}\end{array}$ & $\mathrm{k}$ & $\begin{array}{c}\Delta \tau_{\mathrm{A}} \\
{[\mathrm{MPa}]}\end{array}$ & $\begin{array}{c}\Delta \tau_{\mathrm{A}, 50 \%} \\
{[\mathrm{MPa}]}\end{array}$ & $\mathrm{k}_{0}$ \\
\hline StE460 & 209.8 & 4.3 & 178.6 & 4.9 & 90 & 129.6 & 3 & 100 & 144.0 & 5 & 90 & 129.6 & 3 & 100 & 144.0 & 5 \\
\hline $\mathrm{Al} 6082$ & 86.8 & 6.8 & 77.0 & 6.1 & 32 & 46.0 & 3 & 36 & 51.8 & 5 & 36 & 51.8 & 3 & 36 & 51.8 & 5 \\
\hline
\end{tabular}

Tabella 1: Curve a flessione e torsione utilizzate per calibrare il metodo delle CWM.

Il criterio delle CWM è stato inizialmente utilizzato per stimare la vita a fatica (in termini di tensioni nominali) dei giunti saldati in acciaio sollecitati ad ampiezza costante, calibrando il criterio stesso con i dati sperimentali uniassiali ed a torsione riportati in Tab. 1. Le costanti di calibrazione sono invece elencate in Tab. 2.

Il confronto fra durata sperimentale, $\mathrm{N}_{\mathrm{f}}$, e durata stimata, $\mathrm{N}_{\mathrm{f}, \mathrm{e}}$, mostrato in Fig. 5 a conferma che, assumendo un valore di $\rho_{\mathrm{w}, \text { lim }}$ pari a 1.4, le stime fornite dal metodo delle CWM per sollecitazioni biassiali ad ampiezza costante cadono all'interno delle banda di dispersione delle due curve (uniassiale e torsionale) utilizzate per calibrare il metodo stesso.
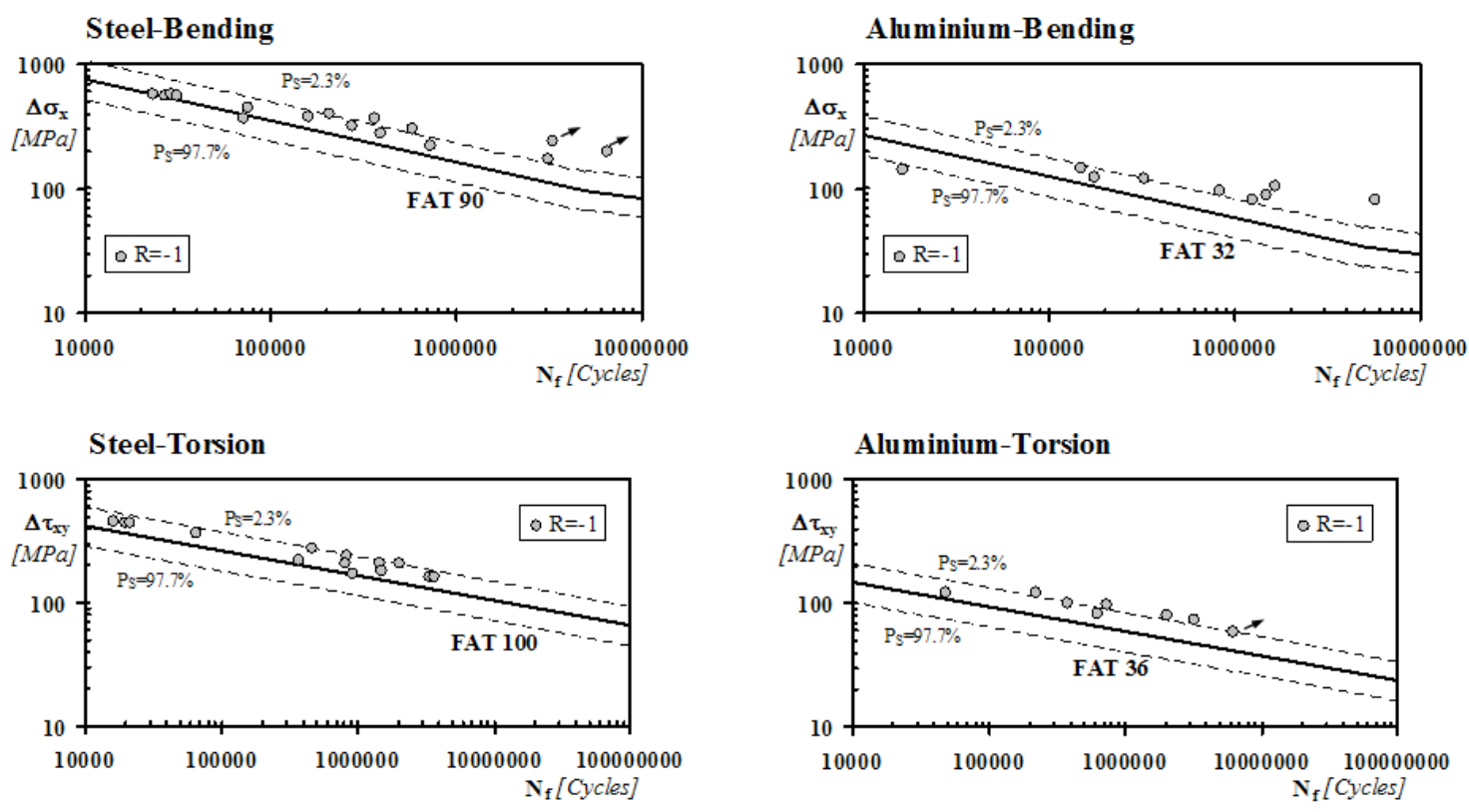

Figura 4: Confronto fra le curve di normativa IIW [10] ed i risultati sperimentali di $[5,6]$.

Per quanto concerne invece i risultati ottenuti con sollecitazioni ad ampiezza variabile con spettro gaussiano, il metodo delle CWM è stato applicato nell'ipotesi (come suggerito in [5]) di un valore pari a 0.35 per il danno critico nella somma di Palmgren-Miner. La Fig. 5b mostra chiaramente che, per sollecitazioni biassiali ad ampiezza variabile, il metodo proposto, basato sulle CWM ed il MMV, fornisce stime di durata che cadono all'interno della banda di dispersione definita dalle due curve ad ampiezza costante usate per calibrare il metodo stesso. 


\begin{tabular}{ccccccccccccccc}
\hline Materiale & \multicolumn{3}{c}{ Risultati sperimentali } & \multicolumn{4}{c}{ Tensioni nominali } & \multicolumn{4}{c}{ Tensioni strutturali ('hot-spot') } \\
& $\alpha$ & $\beta$ & $\mathrm{a}$ & $\mathrm{b}$ & $\alpha$ & $\beta$ & $\mathrm{a}$ & $\mathrm{b}$ & $\alpha$ & $\beta$ & $\mathrm{a}$ & $\mathrm{b}$ & $\rho_{\mathrm{w}, \mathrm{lim}}$ \\
& {$[\mathrm{MPa}]$} & {$[\mathrm{MPa}]$} & & & {$[\mathrm{MPa}]$} & {$[\mathrm{MPa}]$} & & & {$[\mathrm{MPa}]$} & {$[\mathrm{MPa}]$} & & & \\
$\mathrm{StE} 460$ & -73.7 & 178.6 & -0.6 & 4.9 & -79.2 & 144.0 & -2 & 5 & -79.2 & 144.0 & -2 & 5 & 1.4 \\
$\mathrm{Al} 6082$ & -33.6 & 77.0 & 0.7 & 6.1 & -28.8 & 51.8 & -2 & 5 & -25.9 & 51.8 & -2 & 5 & 1.4 \\
\hline
\end{tabular}

Tabella 2: Costanti di calibrazione del metodo CWM in accordo a tre differenti approcci.

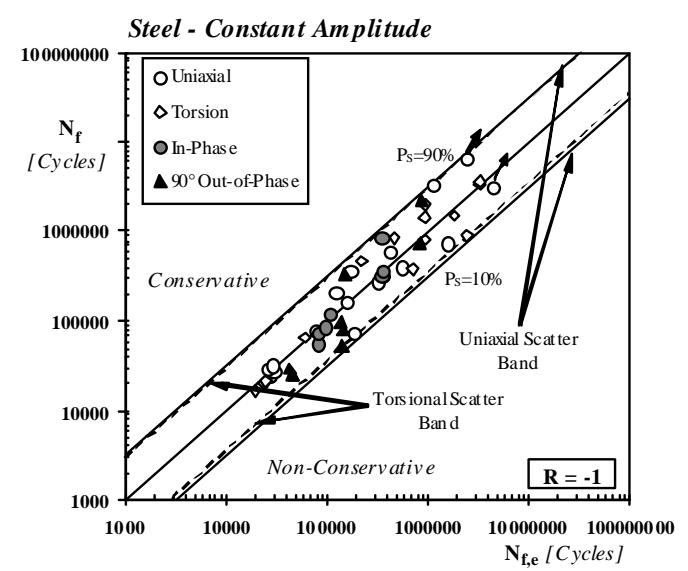

(a)

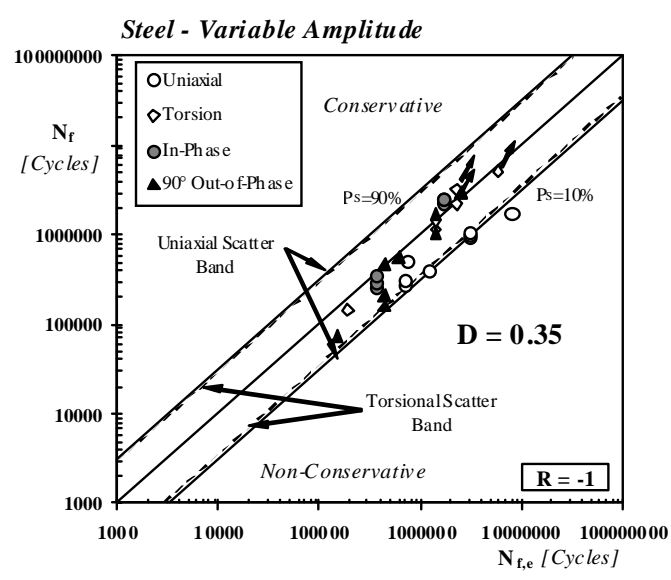

(b)

Figura 5: Stime per sollecitazioni ad ampiezza costante (a) e variabile (b), dove il metodo CWM è calibrato sui dati sperimentali di Tab.1 ed applicato in tensioni nominali.

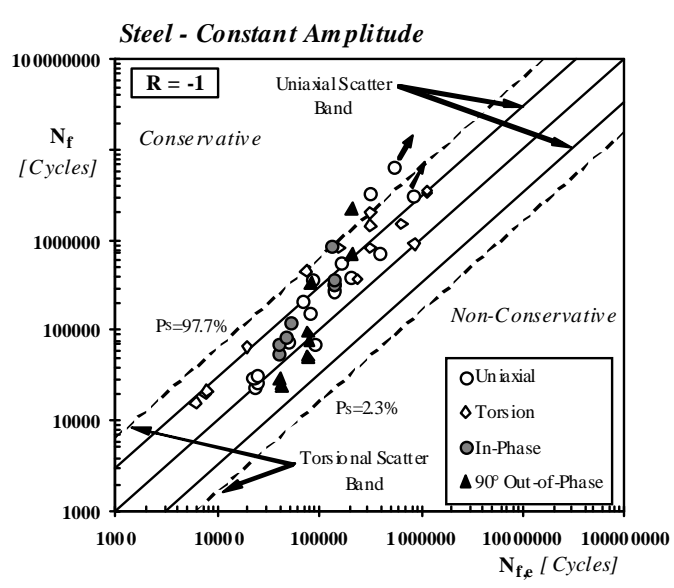

(a)

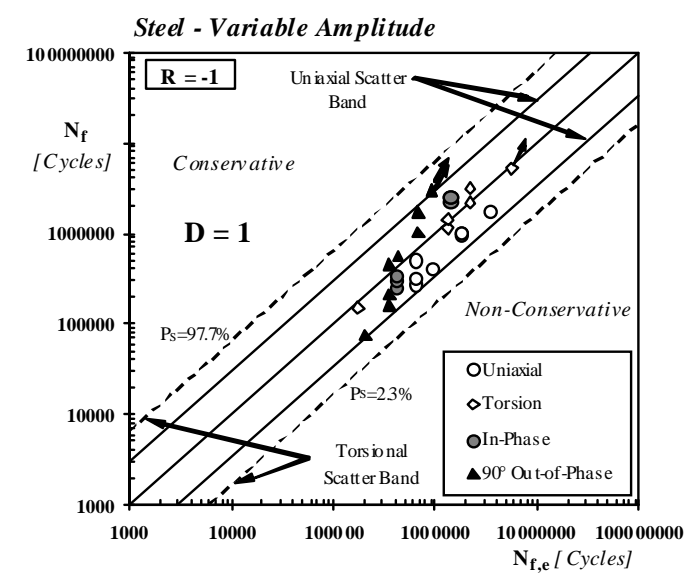

(b)

Figura 6: time per sollecitazioni ad ampiezza costante (a) e variabile (b), dove il metodo CWM è calibrato sulle curve di normativa IIW ed applicato in tensioni nominali.

Per verificare, successivamente, se il metodo proposto fornisce stime di durata in accordo con le normative vigenti, il metodo stesso è stato applicato, ancora in termini di tensioni nominali, al set di dati sperimentali già considerato in precedenza, ma utilizzando le curve di resistenza uniassiale e torsione fornite, per le geometrie adottate, dall'IIW [10]. Si noti che le costanti di calibrazione $(\alpha, \beta, a, b)$ riportate in Tab. 2 sono state ottenute ricalcolando le curve sperimentali di normativa per una probabilità di sopravvivenza, $\mathrm{P}_{\mathrm{s}}$, pari al $50 \%$.

Come confermato in Fig. 6a, il metodo proposto fornisce stime generalmente accurate, in particolare per la zona ad alto numero di cicli, anche quando è calibrato con le curve di normativa. Per quanto concerne infine i risultati per sollecitazioni biassiali con spettro gaussiano, la Fig. $6 \mathrm{~b}$ mostra che, assumendo un valore del danno critico unitario nella sommatoria di Palmgren-Miner, si ottengono stime in buon accordo con i risultati sperimentali. 
I buoni risultati ottenuti considerando giunti saldati in acciaio, ci hanno incoraggiato a provare ad applicare la medesima metodologia anche per la stima della vita a fatica multiassiale di giunti saldati tubo-piastra in lega di alluminio, i cui risultati sperimentali sono peraltro caratterizzati da una maggiore dispersione statistica.

A titolo di esempio, il diagramma di Fig. 7a confronta i risultati sperimentali ad ampiezza variabile con spettro gaussiano con le stime fornite dal metodo CWM applicato in tensioni nominali, dove il metodo stesso è calibrato sui dati sperimentali uniassiali ed a torsione di Tab. 1 (le costanti di calibrazione sono riportate in Tab. 2), e dove si assume, come suggerito in 0, un valore di 0.6 per il danno critico nella sommatoria di Palmgren-Miner.

Per concludere, la Fig. 7b mostra il confronto complessivo fra risultati sperimentali ad ampiezza variabile e le stime fornite dal metodo CWM applicato in termini di tensioni strutturali ('hot-spot'). I risultati sperimentali e le costanti di calibrazione sono riportate in Tab. 1 e Tab. 2, rispettivamente. Come si può osservare, anche nel caso dell'approccio in tensioni strutturali il metodo proposto è in grado di fornire stime accurate, che comunque cadono all'interno delle bande di calibrazione, e questo sia per giunti saldati in acciaio che in lega di alluminio.

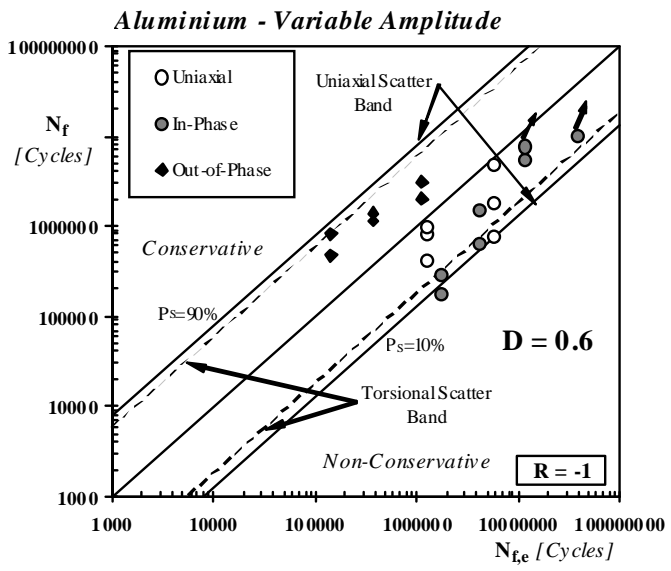

(a)

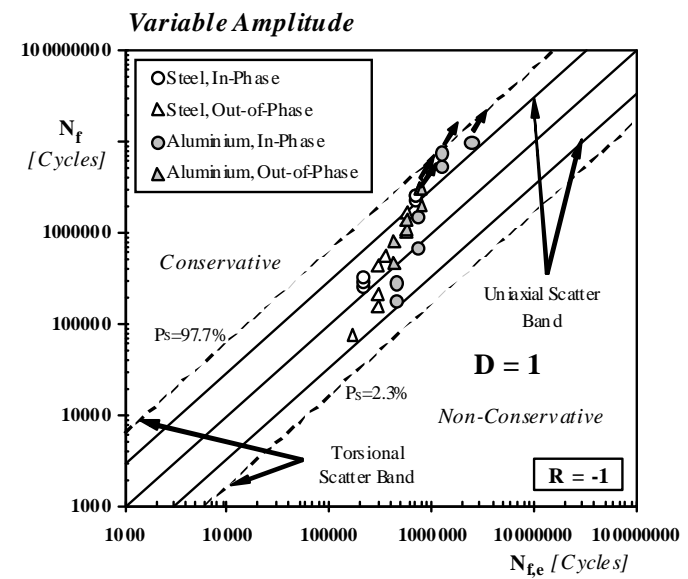

(b)

Figura 7: (a) Stime per sollecitazioni ad ampiezza variabile su giunti in lega di alluminio, in cui il metodo CWM è calibrato sui dati sperimentali di Tab. 1ed applicato in tensioni nominali.

(b) Stime per sollecitazioni ad ampiezza variabile, in termini di tensioni strutturali ('hot-spot').

\section{CONCLUSIONI}

I risultati illustrati nel presente lavoro permettono di trarre le seguenti conclusioni:

1) il metodo delle CWM fornisce stime accurate della durata a fatica di giunzioni saldate in acciaio e lega di alluminio soggette a sollecitazioni multiassiali ad ampiezza variabile;

2) le stime fornite dall'approccio proposto, sia in termini di tensioni nominali che strutturali ('hot-spot'), si sono rivelate in buon accordo con le normative vigenti;

3) è necessario approfondire lo studio del problema della definizione dei cicli in presenza di sollecitazioni multiassiali ad ampiezza variabile allo scopo di formalizzare metodi di conteggio che consentano di stimare il danneggiamento a fatica in modo rigoroso ed univoco.

\section{APPENDICE A: FORMULE ESPLICITE PER SOLLECITAZIONI BIASSIALI}

$\mathrm{P}$

er una sollecitazione biassiale $\sigma_{\mathrm{x}}(\mathrm{t}), \tau_{\mathrm{xy}}(\mathrm{t})$, il piano critico è sempre perpendicolare al piano x-y in Fig. $2\left(\theta=90^{\circ}\right.$, $\left.\xi=0^{\circ}\right)$, mentre la tensione tangenziale risolta dipende solo da $\phi$ :

$$
\tau_{\mathrm{m}}(\mathrm{t})=\frac{\sin (2 \phi)}{2} \sigma_{\mathrm{x}}(\mathrm{t})-\cos (2 \phi) \tau_{\mathrm{xy}}(\mathrm{t})
$$


Analogamente, anche la varianza di $\tau_{\mathrm{m}}(\mathrm{t})$ dipende solo da $\phi$ :

$$
\operatorname{Var}\left[\tau_{\mathrm{m}}(\mathrm{t})\right]=\frac{\sin (2 \phi)^{2}}{4} \mathrm{~V}_{\sigma}+\cos (2 \phi)^{2} \mathrm{~V}_{\tau}-\sin (2 \phi) \cos (2 \phi) \mathrm{C}_{\sigma, \tau}
$$

dove $V_{\sigma}=\operatorname{Var}\left[\sigma_{x}(t)\right], V_{\tau}=\operatorname{Var}\left[\tau_{x y}(t)\right]$ e $C_{\sigma, \tau}=\operatorname{Cov}\left[\sigma_{x}(t), \tau_{x y}(t)\right]$. Uguagliando a zero la derivata prima di Eq. (A.2), si ottengono le direzioni di massima varianza per $\tau_{\mathrm{m}}(\mathrm{t})$, Tab. 3 .

Nel caso particolare di sollecitazioni biassiali sinusoidali sincrone sfasate ( $\delta$ è lo sfasamento):

$$
\begin{aligned}
& \sigma_{x}(t)=\sigma_{x, a} \sin (\omega t) \\
& \tau_{x y}(t)=\tau_{x y, a} \sin (\omega t+\delta)
\end{aligned}
$$

con $\mathrm{V}_{\sigma}=\sigma_{\mathrm{x}, \mathrm{a}}^{2} / 2, \mathrm{~V}_{\tau}=\tau_{\mathrm{xy}, \mathrm{a}}^{2} / 2, \mathrm{C}_{\sigma, \tau}=\sigma_{\mathrm{x}, \mathrm{a}} \tau_{\mathrm{xy}, \mathrm{a}} \cos (\delta) / 2$, le direzioni di massima varianza sono ottenibili utilizzando $\mathrm{i}$ risultati di Tab. 3, posto $C_{\sigma, \tau}=\delta_{x, x y}\left(\delta_{x, x y}=\pi / 2, \delta_{x, x y} \neq \pi / 2\right)$ e:

$$
\begin{array}{ccc}
\left(\mathrm{V}_{\sigma}-4 \mathrm{~V}_{\tau}\right)=\left(\sigma_{\mathrm{x}, \mathrm{a}}-2 \tau_{\mathrm{xy}, \mathrm{a}}\right) ; \phi_{0}=\frac{1}{4} \arctan \left(\frac{4 \sigma_{\mathrm{x}, \mathrm{a}} \tau_{\mathrm{xy}, \mathrm{a}} \cos \delta}{\sigma_{\mathrm{x}, \mathrm{a}}^{2}-4 \tau_{\mathrm{xy}, \mathrm{a}}^{2}}\right) & \\
\hline\left(V_{\sigma}-4 V_{\tau}\right)<0 & \mathrm{C}_{\sigma, \tau} \neq 0 & \mathrm{C}_{\sigma, \tau}=0 \\
\left(V_{\sigma}-4 V_{\tau}\right)=0 & \frac{3 \pi}{8}+i \frac{\pi}{2} & \text { qualunque } \phi \\
\left(V_{\sigma}-4 V_{\tau}\right)>0 & \frac{\pi}{4}+i \frac{\pi}{2} \\
\text { dove } \phi_{0}=\frac{1}{4} \arctan \left(\frac{4 C_{\sigma, \tau}}{V_{\sigma}-4 V_{\tau}}\right), \mathrm{i}=0,1,2, \ldots
\end{array}
$$

Tabella 3: Angoli $\phi$ per la direzione di massima varianza per sollecitazioni biassiali

\section{BIBLIOGRAFIA}

[1] D. Radaj, C. M. Sonsino, W. Fricke, Fatigue Assessment of Welded Joints by Local Approaches, Woodhead Publishing Limited, Cambridge, UK (2007).

[2] M. Bäckström, G. Marquis, Fatigue Fract. Engng. Mater. Struct., 24 (2001) 279.

[3] M. Kueppers, C. M. Sonsino, Fatigue Fract. Engng. Mater. Struct. 26 (2003) 507.

[4] C. M. Sonsino, R. Pfohl, Int. J. Fatigue, 12 (1990) 425.

[5] C. M. Sonsino, M. Kueppers, Fatigue Fract. Engng. Mater. Struct., 24 (2001)309.

[6] M. Kueppers, C. M. Sonsino, Int. J. Fatigue, 28 (2006) 540.

[7] C. M. Sonsino, Int. J. Fatigue 31 (2009) 173.

[8] Anon., Design of steel structures. ENV 1993-1-1, EUROCODE 3, (1988).

[9] Anon., Design of aluminium structures - Part 2: Structures susceptible to fatigue. ENV 1999, EUROCODE 9, (1999). [10] A. Hobbacher, Recommendations for fatigue design of welded joints and components. IIW Document XIII-215107/XV-1254-07, (2007).

[11] L. Susmel, R. Tovo, Fatigue Fract. Engng. Mater. Struct., 27 (2004) 1005.

[12] L. Susmel, R. Tovo, Int. J. Fatigue, 28 (2006) 564.

[13] L. Susmel, P. Lazzarin, Fatigue Fract. Engng. Mater. Struct., 25 (2002) 63.

[14] P. Lazzarin, L. Susmel, Fatigue Fract. Engng. Mater. Struct., 26 (2003)1171.

[15] W. Bedkowski, E. Macha, M. Ohnami, M. Sakane, J. Eng. Materials and Technology, 117 (1995) 183.

[16] K. Bel Knani, D. Benasciutti, A. Signorini, R. Tovo, Int. J. Materials Product Tech., 30 (2007) 172. 
[17] L. Susmel, R. Tovo, P. Lazzarin, Int. J. Fatigue, 27 (2005) 928.

[18] S. Sarkani, D. P. Kihl, J. E. Beach, Prob. Engng. Mech., 9 (1994) 179.

[19] D.P. Kihl, S. Sarkani, J.E. Beach, Int. J. Fatigue, 17(5) (1995) 321.

[20] D. Benasciutti, R. Tovo, Int. J. Fatigue, 27 (2005) 867.

[21] D. Benasciutti, R. Tovo, Prob. Engng. Mech., 20 (2005) 115. 